 \\ Data from a Brazilian Public Hospice for Cancer Patients
}

Ayres, BR; Zeni, EM; Neto, RS; Chiba, T.

Background and aim: Hospice care is associated with improved quality of life, healthcare cost reduction and lower rates of complicated grief. Over 100 million people will need palliative care services, yet less than $8 \%$ will have access to them. There is need for discussion regarding the development of the field in Brazil and for spreading the work of our hospices. This study aimed to characterize the patients attended at a public Brazilian hospice for cancer patients.

Methods: Internment information was pulled from electronic records regarding the period between March 1st, 2016 and July 31st, 2018. Data collected concerned gender, age, diagnosis, length of stay, outcome (decease/discharge/evasion). Calculations of average and median were obtained through the online tool Google Sheets.

Results: From a total of 1120 admissions, 1058 resulted in decease, 61 in discharge and 1 evasion. Average stay time was 17.2 days (1 241) and the median was 9 days. 478 admissions $(42.68 \%)$ lasted 7 days or less. 14 patients were admitted twice.

\section{Lenght of Stay}

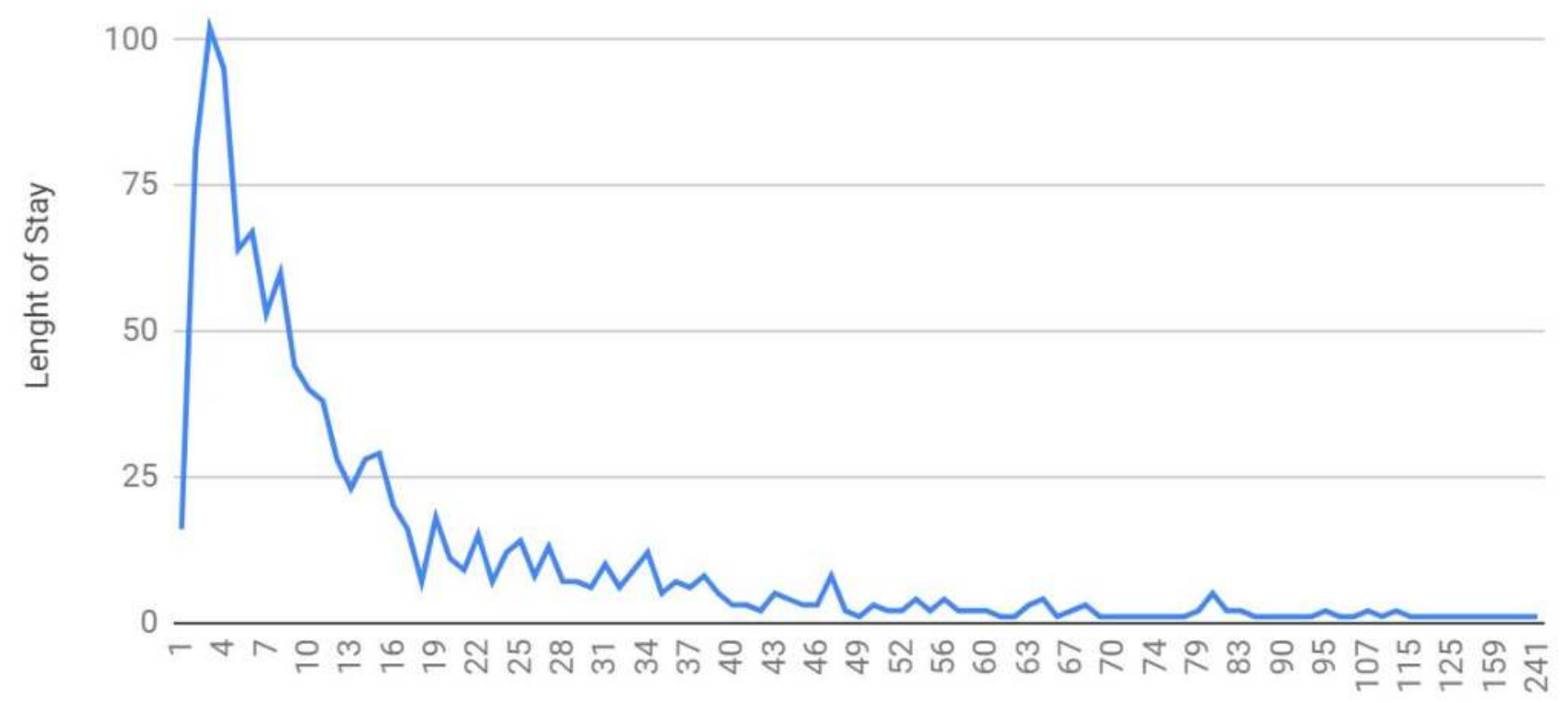

Out of the 1106 patients, 637 were male (57.59\%). Average age was 65.2 years and age median was 65 (19 101). Most prevalent diagnosis were lung cancer (146), head and neck (133), bowel and rectum (110), gastric (80), breast (69), esofagus (63), central nervous system (58) and prostate (55). 52 diagnosis were considered unreliable and excluded.
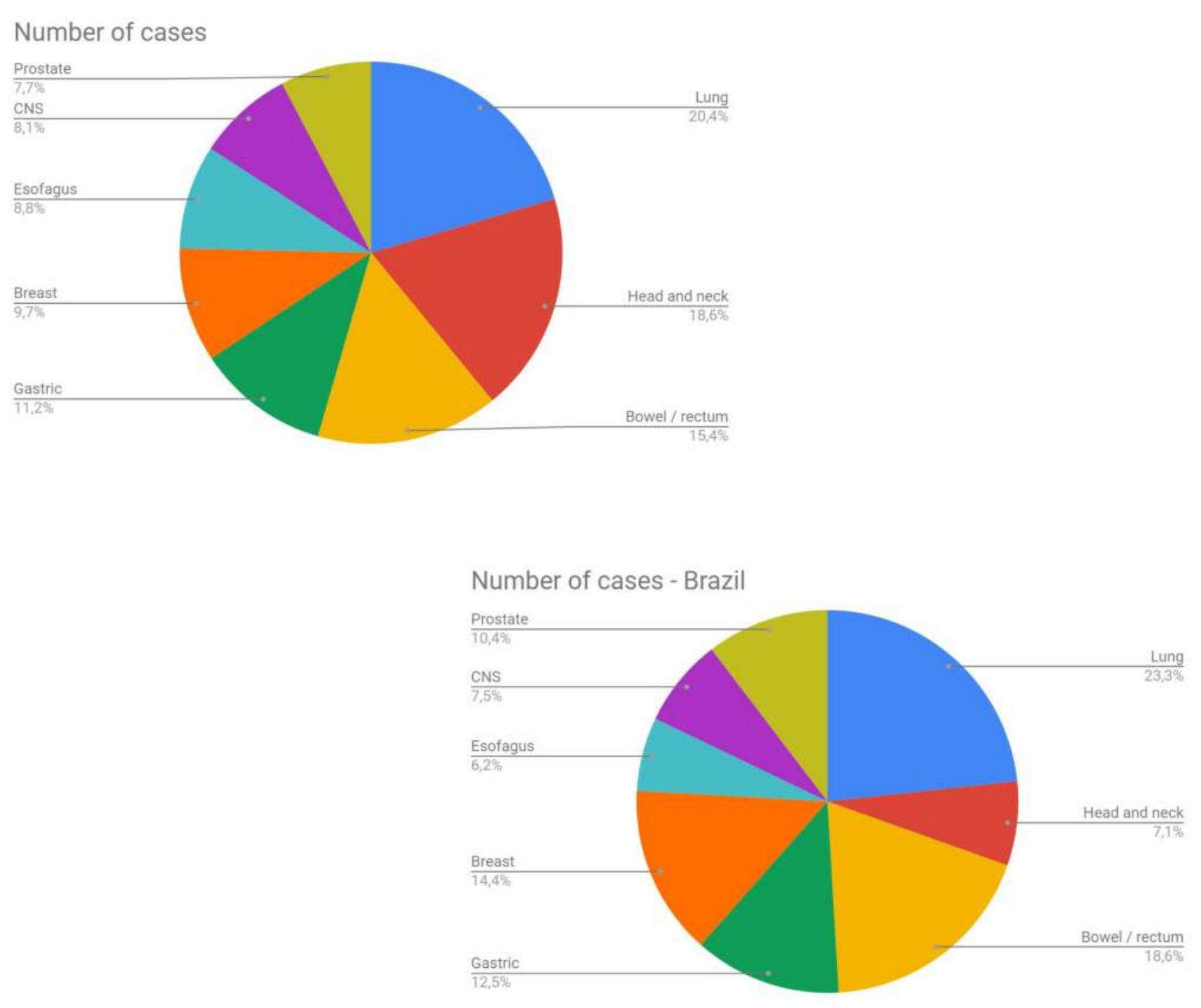

Discussion: Our data collection showed that lung cancer was the most prevalent $(13.79 \%)$, similar to the national data (12.62\% of cancer deaths). There was however a smaller prevalence of frequent neoplasms, such as breast and prostate, and markedly higher of head and neck cancer $(12.57 \%)$ if compared to the national mortality $(3.7 \%)$, suggesting a connection to the higher need for complex care. There was a large number of short admissions, suggesting late referral, with a possible negative impact on care quality. Another impact is the reduced use of a hospice facility as a strategy for health care cost reduction.

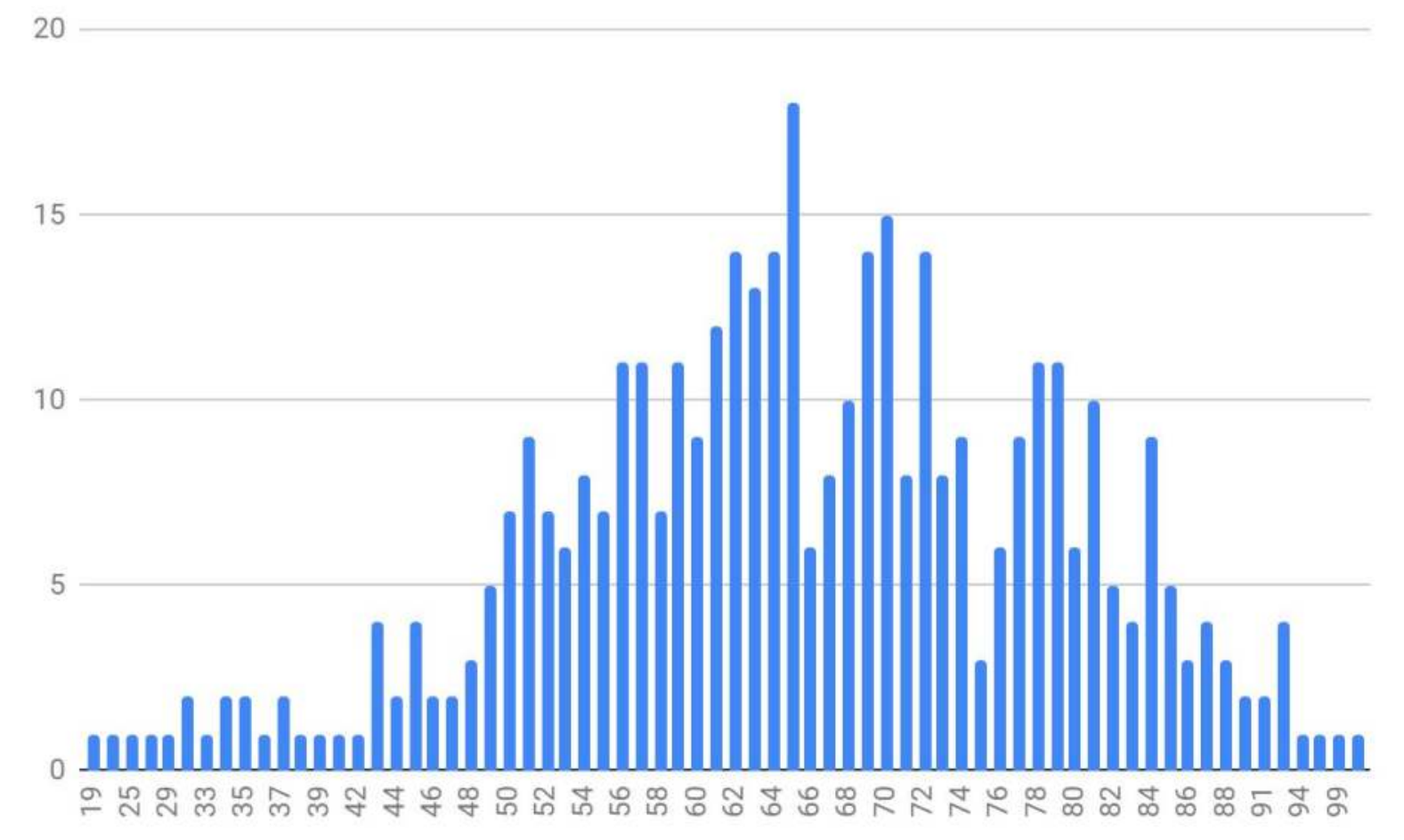

